Edith Cowan University

Research Online

Research outputs 2014 to 2021

2016

Permanent slides for morphological studies of small crustaceans:

Serban's method and its variation applied on Bathynellacea

(Malacostraca)

Giulia Perina

Edith Cowan University / Western Australian Museum

Ana I. Camacho

Museo Nacional de Ciencias Naturales, Spain

Follow this and additional works at: https://ro.ecu.edu.au/ecuworkspost2013

Part of the Terrestrial and Aquatic Ecology Commons

$10.1163 / 15685403-00003576$

This is an Author's Accepted Manuscript of:

Perina, G., \& Camacho. A.I. (2016). Permanent slides for morphological studies of small crustaceans: Serban's method and its variation applied on Bathynellacea (Malacostraca). Crustaceana, 89(10), 1161-1173.

https://brill.com/view/journals/cr/89/10/article-p1161_5.xml

This Journal Article is posted at Research Online.

https://ro.ecu.edu.au/ecuworkspost2013/2273 


\title{
PERMANENT SLIDES FOR MORPHOLOGICAL STUDIES OF SMALL CRUSTACEANS: SERBAN'S METHOD AND ITS VARIATION APPLIED ON BATHYNELLACEA (MALACOSTRACA)
}

BY

\section{G. PERINA ${ }^{1,2}$ ) and A. I. CAMACHO ${ }^{3}$ )}

${ }^{1)}$ Centre for Ecosystem Management, School of Science, Edith Cowan University, 270 Joondalup Dv, Joondalup, WA 6027, Australia

2) Western Australian Museum, Locked Bag 49, Welshpool DC, Western Australia 6986, Australia

3) Museo Nacional de Ciencias Naturales (CSIC), Dpto. Biodiversidad y Biología Evolutiva, C/ José Gutiérrez Abascal 2, 28006-Madrid, Spain

\begin{abstract}
Morphological studies of small invertebrates often involve the preparation of slides to observe minute body parts under a compound microscope. Preparation should facilitate observation, through traditional optic microscopy, of small surface structures on different planes, like pores, spines and setae. Various methods and techniques, using different mounting media that specialists have adopted to observe and preserve small crustaceans, have their advantages and disadvantages.

Within the order Bathynellacea, specimens in the family Bathynellidae are particularly challenging due to their small size ( 0.5 to $2.25 \mathrm{~mm}$ body length) and very delicate exoskeleton, which tends to be completely digested when using common clearing mounting media, making future consultations impossible. Permanent slides are fundamental to preserve small specimens for scientific collections, because temporary slide preparations can easily result in the loss of body parts in the passage between slide and vial and vice versa.

Dr Eugene Serban worked on Bathynellacea for more than 40 years, improving the preparation and preservation of delicate specimens using stained glycerol-jelly and double cover slip mounting technique. His method is described here with a variation that speeds up the original procedure and implemented in most recent years by one of the authors (Camacho). The technique provides excellent preservation and visualisation of body parts on permanent slides, which do not need curation tasks and can last for many years.
\end{abstract}

\section{INTRODUCTION}

There are many methods to prepare invertebrates for morphological studies: temporary, semi-permanent and permanent slides with or without stains or clearing agents 
and using different mounting media (Humes \& Gooding, 1964; Koomen \& Von Vaupel Klein, 1995; Stock \& Von Vaupel Klein, 1996). The whole animal or the dissected body parts of different taxa are mounted and the technique applied depends on the characteristics and the use of the invertebrate mounted (Galigher \& Kozloff, 1971; Smithsonian National Museum of Natural History, 2007). Some taxonomists working on relatively large invertebrates (over 2-3 mm) prefer mounting the whole specimen or its dissected body parts on temporary slides, and successively re-bottle them in ethanol or other preserving liquids (K. M. Abrams, personal communication, February 16, 2015). While this technique can be successful, small invertebrates (less than $2 \mathrm{~mm}$ ) usually require permanent or semipermanent preparations to avoid the loss of specific body parts, and to allow easy consultation, especially in the case of type material, when new species are described.

Several water or organic solvent based mounting media can isolate and protect specimens from the atmosphere, and ideally should be transparent, resistant to contamination by fungi or bacteria, and set without crystallizing or shrinking to facilitate preservation and observation with a compound microscope (Ravikumar, Surekha, \& Thavarajah, 2014). Euparal, Canadian Balsam, and Eukitt are popular water-insoluble mounting media for invertebrates and have different advantages and disadvantages. The main issue with such liquids is that the specimen, or its body parts, cannot be retrieved if the slide is ruined and moreover, details are difficult to see (Labwork, 2010). The commonly used water-soluble mounting media (Hoyer; Faure; lactic acid and lactophenol) are also used as clearing agents and work very well on organisms with thick and robust exoskeletons, digesting soft tissues and enhancing cuticle structures (Faraji \& Bakker, 2008; Harvey, 2011; I. Karanovic, Eberhard, \& Perina, 2012; T. Karanovic \& Cooper, 2012). However, one of their disadvantages is that they keep digesting the body parts to a point that it is hard to find the structures on the slide (Smithsonian National Museum of Natural History, 2007). Other common aqueous mounting media are: glycerine (or glycerol $\mathrm{C}_{3} \mathrm{H}_{8} \mathrm{O}_{3}$ ) and glycerol jelly. They require anti-fungal or bacteriostatic agents like phenol or thymol to prevent the growth of undesired organisms. If glycerine and glycerol jelly are used for long term preparations, sealing the edges of the cover slip with nail polish or paraffin and Araldite (Ranga Reddy, Shaik, \& Totakura, 2015) is necessary to avoid contact with air which can damage the specimen (Camacho \& Puch, 1990; Ravikumar et al., 2014). The seal should be re-laid periodically since the nail polish tends to shrink and crack. These methods seem to be successful with small, fragile taxa like Bathynellacea (Camacho \& Puch, 1990; Cho \& Humphreys, 2010; Ranga Reddy, 2002), but the curation of such collections is not convenient, especially for numerous slides.

One of the authors (Camacho) trained (1993-1995) on Syncarida slide preparation under a world specialist for that group, Dr Eugene Serban. Thanks to his effort in developing a good dissecting and mounting method for small and fragile specimens, we have wellpreserved holotypes and type series of 42 species that he described between 1963 and 1998 (Camacho, unpublished observation). The method is based on the classic use of Kaiser glycerine jelly but with three adaptations: 1 . the jelly is stained with methylene blue; 2 . two 
cover slips are used instead of a slide; 3 . special metal slides are used to support the cover slips.

Camacho has been using Serban's method for more than 15 years (and has prepared more than 2000 slides) and recently improved the method using paraffin as it is applied in Nematoda slides (De Ley, 1995) (Covacho personal communication, 2013). This refinement reduces preparation time and avoids curation tasks. The methods described here were specifically designed for Bathynellacea specimens, but they can also be applied to other taxa.

\section{PREPARATION, DISSECTION AND MOUNTING}

Serban's method

It is an adaptation of the classic method that uses glycerine jelly prepared according to Kaiser (1880). Few years ago, one of the main difficulties was preparing the jelly with the correct density, but today it is currently available on the market.

Step one: passage to $100 \%$ glycerine. - Place specimen, usually preserved in ethanol, on a concave slide or watch glass (fig. 1A-1B) in a large drop of 50:50 100\% ethanol and bidistilled glycerine (glycerol $\mathrm{C}_{3} \mathrm{H}_{8} \mathrm{O}_{3}$ ). Leave it for about 24 hours to achieve an isotonic state through ethanol evaporation. Each sample is labelled and covered to avoid dust collection.

Step two: selection of the specimen to dissect in $100 \%$ glycerine. - The bathynellacean is placed in a small drop of pure bidistilled glycerine, measured under a compound microscope and successively dissected, starting from the pleotelson. All appendages are separated from the rest of the body segments during the dissection. Each body part is positioned in a small drop of glycerine making sure to maintain the order of the thoracopods (example fig. 1C).

Step three: inclusion of body parts in stained glycerine jelly. - The different body parts are then moved, with a micro-pin, to a clean $24 \times 24 \mathrm{~mm}$ cover slip, maintaining the thoracopods order (example fig. 1C). Fix each cover slip on a slide, the authors use a small amount of modelling clay on the corners, (fig. 1D) to work easily and to record the details of the specimen on one side of the slide. Keep cover slips in ethanol 70 or $100 \%$ to avoid static and dirt/dust collection. Dry them with a tissue before use.

Melt the glycerine jelly with the methylene blue in bain-marie (the amount of methylene blue depends on the quantity of jelly used; in general, add a small amount (fig. $1 E$ ), mix well and add more until the melted jelly assumes a strong blue colour. Keep the jelly in hot water to maintain it as a fluid. With a loop-needle, put a small drop of "blue jelly" near each body part previously placed on the cover slip. With a micro needle push each limb into the jelly and displace the liquid to position the body part flat and have a thinner layer of jelly (fig. 1F). Clean the loop-needle and the micro needle each time to keep the preparation dust free. Once all the body parts are in blue glycerine jelly, cover the slide to avoid dirt collection and leave it for 24 hours so the jelly solidifies.

Step four: total inclusion in glycerine jelly. - To be sure the jelly is completely dehydrated, pass the slide over a flame for few seconds. Afterwards, with a warm pipette 
(heated previously with hot water), lay another stratum of blue glycerine jelly warm enough to be fluid, but not too hot to melt the solidified jelly drop laid previously and displace the body parts. Cover all the body parts making sure they do not move from their flat position (fig. 1G). Cap the cover slip gently with an $18 \times 18 \mathrm{~mm}$ clean cover slip (fig. 1H). Apply gentle pressure using a needle to displace excess jelly and any air bubbles trapped under the cover slip. Refrigerate the preparation for 10-15 minutes, making sure it is kept horizontal. Subsequently, cut the edges of jelly that emerge from the coverslip and repeat the operation after a few hours, so that any jelly which flows out of the cover slips can be removed. Allow the slide to sit horizontal for at least 24 hours, so the glycerine jelly can solidify and the surplus can overflow, leaving the preparation at the appropriate thickness for microscopy.

Step five: mounting coverslips on metal support. - After 24 hours, cut the edges of the jelly that emerge from the coverslip and clean it with a tissue soaked in water and soap. Seal the "sandwich-slip" twice with nail polish. Add methylene blue to the nail polish to avoid discoloration of the preparation. Mount the slip on a metal slide and label it (fig. 1l). The preparation is permanent, but it may be possible to adjust some setae by carefully warming the "sandwich-slip" on a flame and gently manipulating the top slide with tweezers or a tip while observing the process under a stereo microscope ensuring the body parts do not move.

It is best to let the preparation sit for another day to ensure the glycerine jelly solidifies completely and the body parts do not move when observed with the $100 x$ oil immersion objective.

For big or very dirty specimens, lactotophenol can be added to the drop of glycerine when the animal is dissected to clear and clean the tissues.

\section{Serban's method modified by Camacho}

Step one and two as above.

Step three: paraffin cover slip preparation. - Melt pure histological paraffin and prepare one cover slip with a thin layer of paraffin thus creating a lattice with empty spaces to position the body parts. The lattice can be created by immersing a custom made tool (fig. $2 A-2 B$ ) in liquid paraffin (melted on a hot plate), then placing it on a cover slip to deposit a thin layer of paraffin (fig. 2C-2D-2E). With a looped wire lay the two horizontal lines of paraffin creating 12 empty cells (fig. 2F-2G-2H) If the amount of paraffin is excessive, it will overflow once melted, and will cover the outside part of your cover slide (which can be scraped with a blade and cleaned of paraffin) while if it is insufficient, the paraffin will not seal the preparation completely. To work easily with cover slips and to record the details of the specimen, fix each cover slip on a slide using a small amount of modelling clay on the corners (figs. 1D-2E).

Step four: placement of body parts on cover slip and inclusion in glycerine jelly. - The different body parts are moved with a micro-pin from the glycerine to the cells created with 
the paraffin on the cover slip, thus maintaining the order of thoracopods. The inclusion of the specimen in stained glycerine jelly follows step three of Serban's method (fig. 3A-3B).

Step five: inclusion in paraffin. - After the jelly has solidified (about 24 hours), lay a clean cover slip (24×24 mm) on it and melt the paraffin on a hot plate for few seconds (fig. 3C-3D). The paraffin will fill all the gaps around the jelly and the air will escape from under the cover slip sealing the "sandwich-slip". Do not leave the slide too long otherwise the jelly will melt too, and the paraffin will flow outside the slips. Let the whole preparation sit for few hours before mounting it on a metal slide (fig. 3E-3F). 


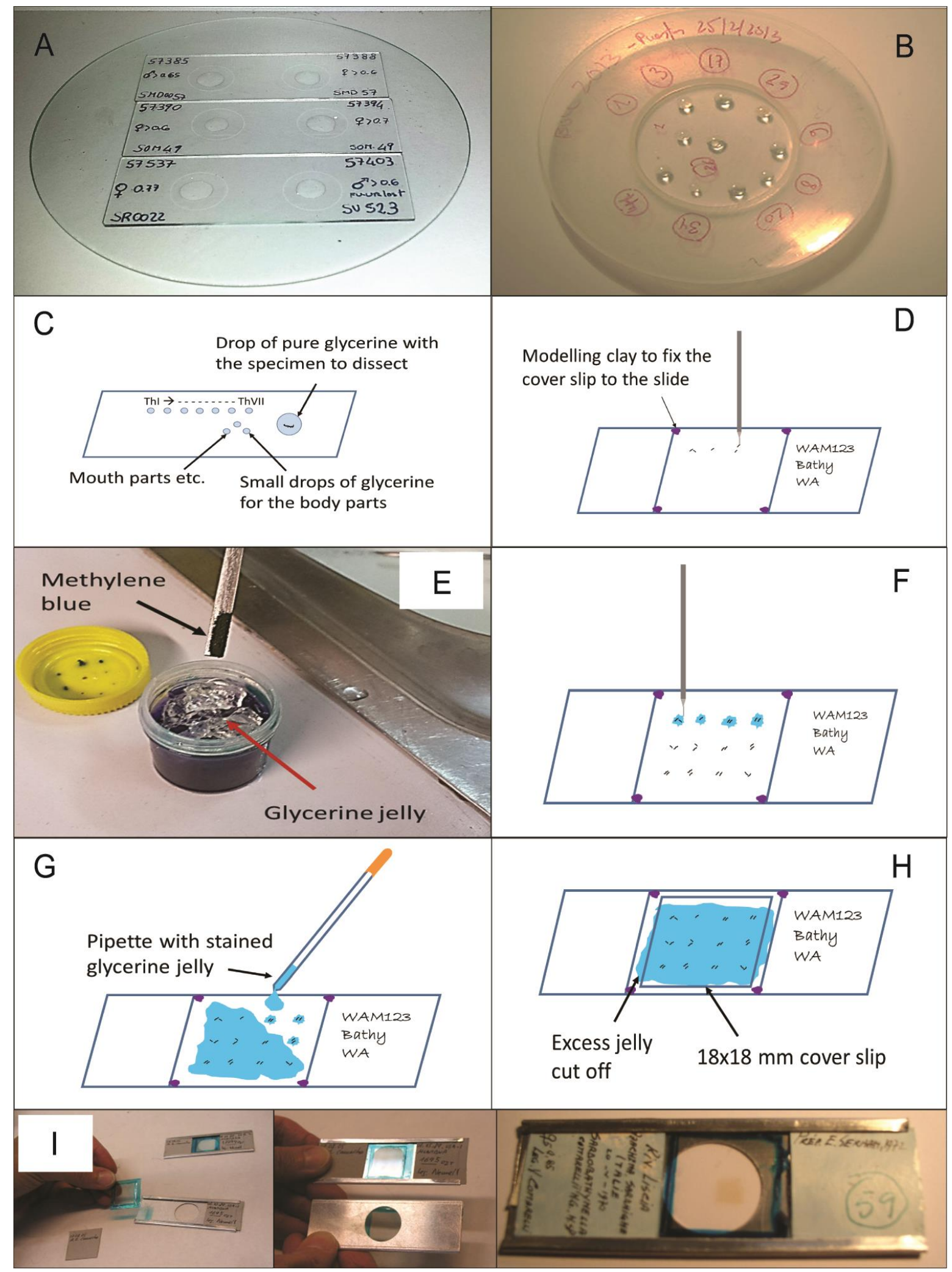

Figure 1. Dissection and permanent preparation of Bathynellidae, Serban's method. A-B: specimens in 50:50 ethanol $100 \%$ and bidistilled glycerine (concave slide and watch glass). C: dissection of a bathynellid specimen in glycerol. D: placement of the different body parts on a coverslip fixed on a slide. E: glycerine jelly stained with methylene blue preparation. I: C example of Serban's slide mounted on metal support. 


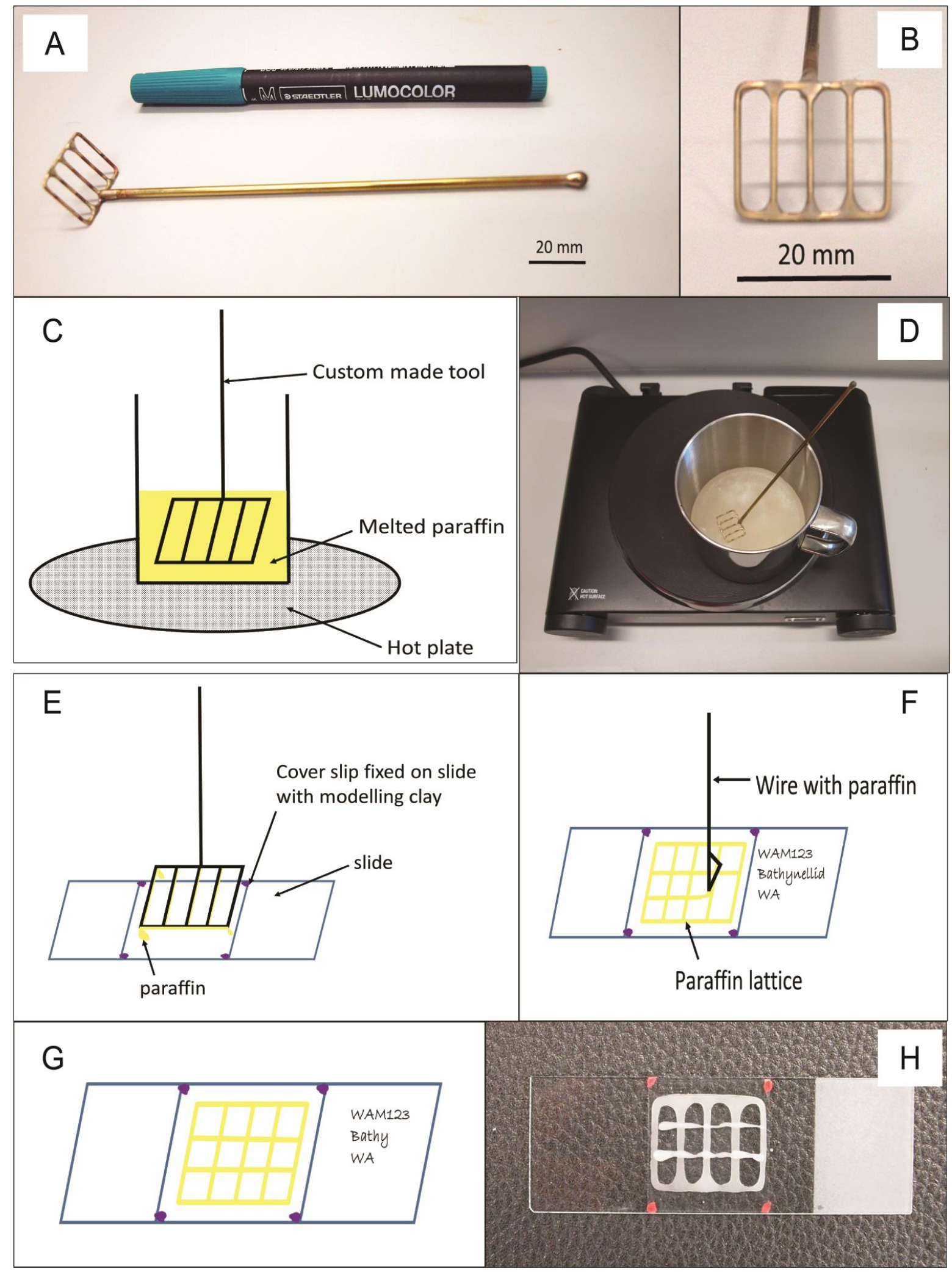

Figure 2. Permanent preparation of Bathynellidae, Camacho's variation implemented. A-B: custom made tool to create the paraffin lattice on cover slip. The surface needs to be flat and smooth. C-D: custom made tool in the melted paraffin. E: paraffin lattice on cover slip preparation. F: paraffin lattice completion through a looped wire. G-H: paraffin lattice on cover slip. 


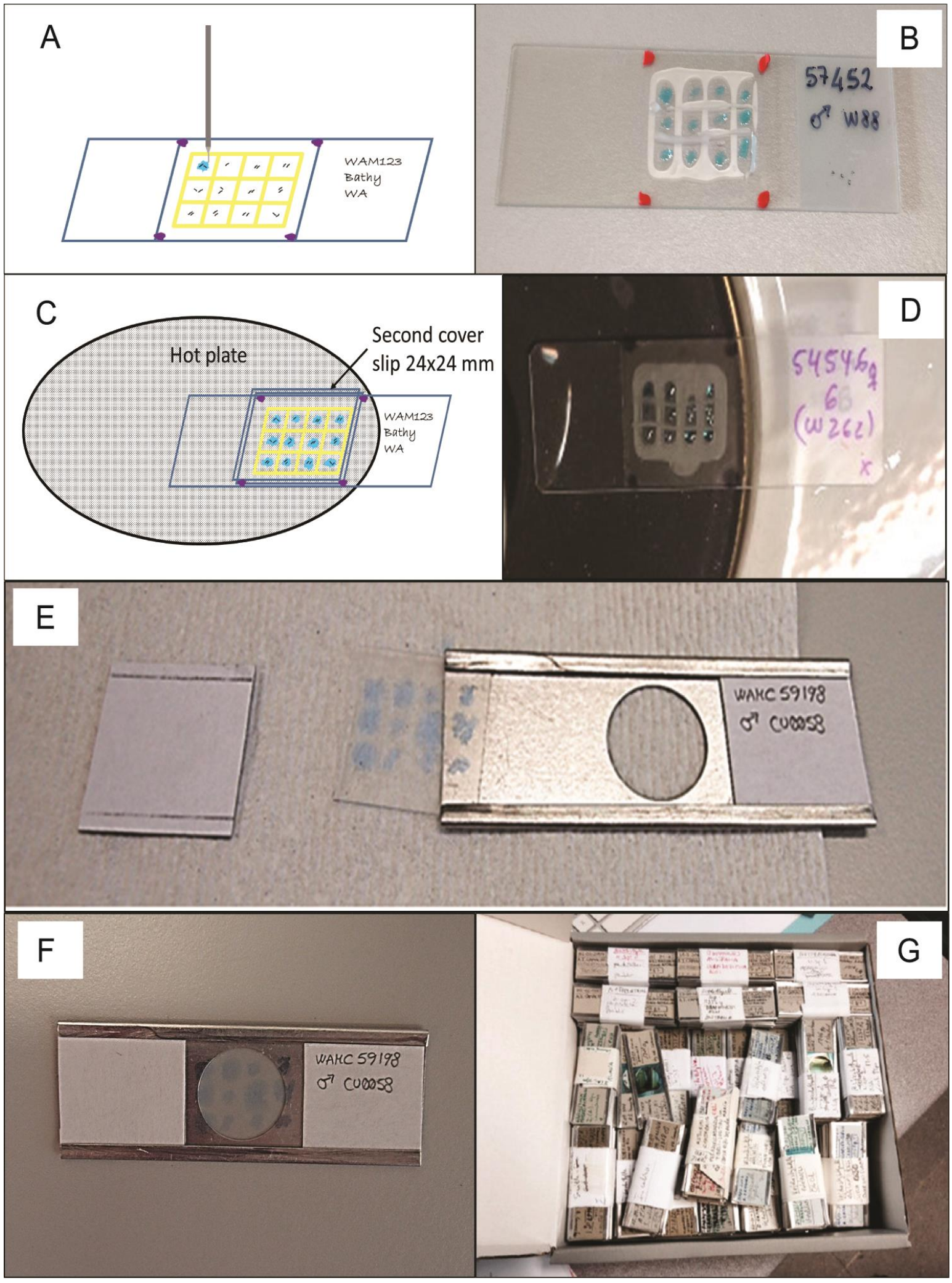

Figure 3. Permanent preparation of Bathynellidae, Camacho's variation implemented (continue). AB: body parts inclusion in stained glycerine jelly and flatten spreading the jelly with a needle. CD: paraffin lattice melting on a hot plate after layering the second cover slip on top. E: "sandwich cover slip slide" slotted in a metal slide. F-G: final slide and box with slides for easy transport. 


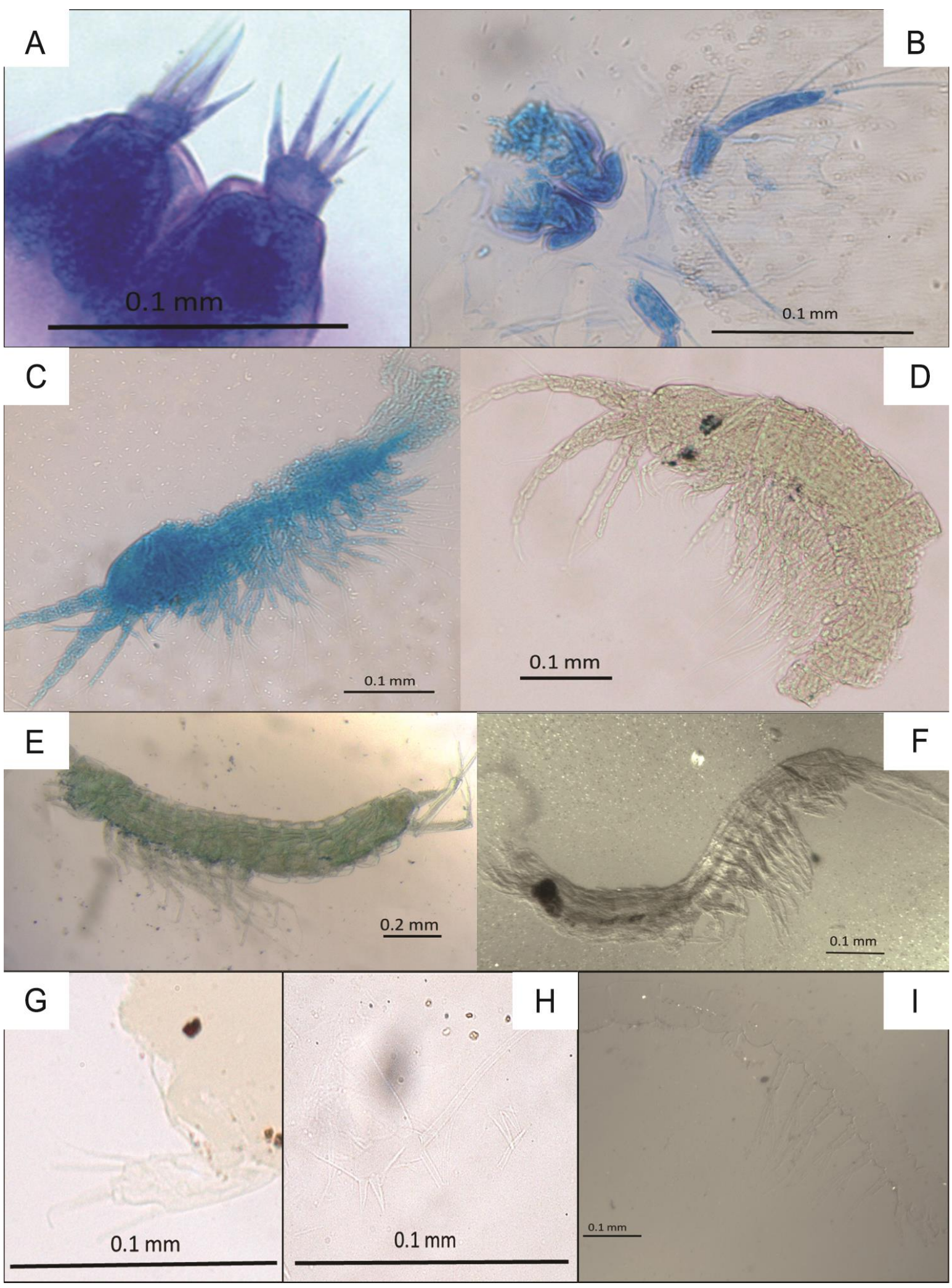

Figure 4. Images of Bathynellacea prepared with different methods. A-B-C: bathynellid furca, male thoracopod VIII and pleopod, half body in stained glycerine jelly (well impregnated tissues). D: bathynellid in stained glycerine jelly: example of poorly impregnated tissues. E: Parabathynellie specimen: Serban's preparation (1993). F: Bathynellidae specimen: E. Serban preparation (1968). G-H: bathynellids body parts: Hoyer preparation (2011). I: parabathynellidae specimen: E. Ortiz Hoyer preparation (1968). 


\section{DISCUSSION}

Advantages of the techniques described

Serban's technique (including Camacho improvements) allows all body parts to be kept on one slide (which can save time during observation, efficiently uses space and lowers the possibility of mistakes), and maintains the order of the appendages. Tissues in stained jelly absorb the methylene blue, become more visible and are easy to find on the slide (fig. 4A4B-4C); although some old specimens do not assimilate much colour (fig. 4D). The inclusion in double slips allows the observation of the specimens from both sides, to reveal morphological structures better, especially when the group studied has complicated 3D features that need drawings from different sides, like the male thoracopod VIII of Bathynellacea. The slides resulting from both methods are quite resistant since the "sandwich slip" is slotted into a metal slide, which does not break if it falls, and can be easily transported (figs. 1I-3E-3F-3G).

Slides can be preserved well in scientific collections for years, which is particularly important in the case of type series.

The paraffin improvement to the method requires a minimum amount of glycerine jelly (which can be quite expensive); saves time (there is no need to wait over 2 days for the jelly to overflow and obtain the right slide thickness), and avoids sealing/re-sealing over time. This is particularly useful with numerous slides, and when preparing type material.

Other advantages are: the glycerine jelly and the paraffin can be purchased (avoiding the preparation of homemade mounting media that could result in slight differences each time), and they do not contain major poisonous substances (although the glycerine jelly on the market does contain a very small amount of phenol, but the jelly used with the paraffin method is minimal).

Very delicate specimens like bathynellids can be preserved for a long time and the quality of the slides should remain excellent for many years. Serban's preparations are still in good condition, even after more than 40 years (fig. 4E-4F), while bathynellids mounted on Faure, Hoyer, Canadian Balsam or other clearing liquids are often very hard to see, even after just a few years (fig. 4G-4H-4I).

Other taxa can be successfully preserved with these methods, especially invertebrates without or with very thin exoskeleton. One of the authors (Camacho) applied these techniques to nematodes and copepods with good results. The shape of the custom made tool to lay paraffin can vary according to the number of appendages that need to be dissected. Different or no staining chemicals can be added to the jelly, providing that they do not have expiry date. Lactophenol can be combined with glycerine when the animal is dissected to clear and clean the tissues. This could be particularly useful for those invertebrates with thick cuticle that need muscles digestion to observe the details.

\section{Disadvantages}

Serban's original method is very time consuming. The paraffin modification can save time, but the jelly drops do need to sit and dry for about 24 hours. However, this 
improvement will save time in the future by avoiding sealing and re-sealing of the slides. Additional issues are: the possible loss of body parts during the passage from glycerine to cover slip, and the paraffin intrusion into the jelly, which can completely or partially cover the body parts. This can be prevented by avoiding contact between glycerine-jelly and paraffin, and melting the paraffin between cover slips at low temperature (if the hot plate is too warm, the paraffin will melt abruptly causing intrusions into the glycerine jelly).

If the preparation is not satisfactory, using Serban's method, it is always possible to separate the two cover slips by warming them up, while it is not possible if the slips are sealed with paraffin.

The construction of the tool needed to create the paraffin lattice (fig. 2A-2B) can be rather challenging: the metal rod must be very fine (diameter of about $1 \mathrm{~mm}$ ); the welding should be very smooth without imperfections and resistant to the paraffin melting temperature $\left(56-60^{\circ} \mathrm{C}\right)$; and the whole tool has to be perfectly horizontal and flat so it can lay a thin stratum of paraffin. The authors used: $1 \mathrm{~mm}$ solid brass or copper rod with joints brazed together with $45 \%$ silver solder.

\section{CONCLUSION}

The morphological study of small crustaceans is often quite challenging, especially in bathynellids, considering their minute size and fragile exoskeleton.

With more than 30 years of research focused on Bathynellacea, Serban had the opportunity to design and refine a specific method to prepare and preserve small and delicate crustaceans. With this paper we recognize his effort and scientific contribution, in particular to the study of the Bathynellidae family, and share our experience with other taxonomists. Although it is quite time consuming, the method we described has many advantages: good conservation of the specimen for a long period (at least 40 years, which is more than other techniques showed); the whole dissected animal is kept on one slide maintaining the order of the appendages and saving space and material; and the "sandwich cover slip" allows the observation, through a compound microscope, from both sides, which helps the study of details.

The improvement implemented by Camacho saves a substantial amount of time and it is, therefore, the preferred technique adopted by the authors. Although these techniques were specifically designed for Bathynellacea, they can be apply to other taxa with success.

Imaging and 3D scanning techniques are improving considerably, however, the preparation and preservation of small dissected invertebrates to study their morphological features are still necessary for taxonomic work and specimen conservation in scientific collections.

\section{ACKNOWLEDGMENT}

This work was supported by: CGL2015-66571-P project (MINECO, Ministerio de Economía y Competitividad, Spain; Australian Government's Australian Biological Resources Study (ABRS) National Taxonomy Research Grant Program (NTRGP); Edith Cowan University 
and Western Australia Museum funding; and International Conference on Subterranean Biology Grant. Many thanks to Carlos Puch and Nicholas Newton for building the tool to lay paraffin and their technical support. Thanks to Tim Newton, Joel Huey, Annette Koender and Greg Maguire for revising the manuscript.

\section{REFERENCES}

CAMACHO, A. I. \& C. PUCH, 1990. Une méthode pour la réalisation de dissections et de préparations provisoires de petits crustacés aquatiques souterrains et interstitiels. Crustaceana, 59(1): 1-8.

CHO, J.-L. \& W. F. HUMPHREYS, 2010. Ten new species of the genus Brevisomabathynella Cho, Park and Ranga Reddy, 2006 (Malacostraca, Bathynellacea, Parabathynellidae) from Western Australia. Journal of Natural History, 44(17-18): 993-1079.

DE LEY, P., 1995. WORMWOOD - A resource for nematode phylogeny. from http://xyala.cap.ed.ac.uk/research/nematodes/fgn/worm/extrafix.html\#tempotop

FARAJI, F. \& F. BAKKER, 2008. A modified method for clearing, staining and mounting plantinhabiting mites. European Journal of Entomology, 105(4): 793-795.

GALIGHER, A. E. \& E. N. KOZLOFF, 1971. Essentials of practical microtechnique ( $2 \mathrm{~d}$ ed.). Lea \& Febiger, Philadelphia.

HARVEY, M. S., 2011. Notes on some Old World schizomids of the genera Ovozomus and Schizomus (Schizomida: Hubbardiidae). Records of the Western Australian Museum, 26: 202 - 208.

HUMES, A. G. \& R. U. GOODING, 1964. A method for studying the external anatomy of copepods. Crustaceana, 6: 238-240. doi: 10.1163/156854064X00650.

KAISER, 1880. Botanisches Zentralblatt (Vol. 1: 25).

KARANOVIC, I., S. M. EBERHARD, \& G. PERINA, 2012. Austromesocypris bluffensis sp. $\mathrm{n}$. (Crustacea, Ostracoda, Cypridoidea, Scottiinae) from subterranean aquatic habitats in Tasmania, with a key to world species of the subfamily. Zookeys, 215: 1-31. doi: 10.3897/zookeys.215.2987.

KARANOVIC, T. \& S. J. B. COOPER, 2012. Explosive radiation of the genus Schizopera on a small subterranean island in Western Australia (Copepoda: Harpacticoida): unravelling the cases of cryptic speciation, size differentiation and multiple invasions. Invertebrate Systematics, 26(2): 115-192.

KOOMEN, P. \& J. C. VON VAUPEL KLEIN, 1995. The Suitability of Various Mounting Media for Permanent Mounts of Small Chitinous Crustaceans, With Special Reference To the Observation of Integumental Organs. Crustaceana, 68(4): 428-437.

LABWORK, 2010. An overview of mounting media for microscopy. from http://www.microbehunter.com/an-overview-of-mounting-media-for-microscopy/

RANGA REDDY, Y., 2002. Habrobathynella nagarjunai $n$. sp., the second representative of Bathynellacea (Crustacea, Syncarida) from groundwaters of South India. Hydrobiologia, 470: 37-43. 
RANGA REDDY, Y., S. SHAIK \& V. R. TOTAKURA, 2015. Camachobathynella meghalayaensis n.gen., n. sp., the first palearctic element of Bathynellacea (Eumalacostraca:

Bathynellidae) from Northeastern India. Journal of Crustacean Biology, 00(0): 1-14. doi:10.1163/1937240X-00002355.

RAVIKUMAR, S., R. SUREKHA \& R. THAVARAJAH, 2014. Mounting media: An overview (Vol. 3).

SMITHSONIAN NATIONAL MUSEUM OF NATURAL HISTORY, 2007. The World of Copepods. from http://invertebrates.si.edu/copepod/techniques.htm\#microscopic

STOCK, J. H. \& J. C. VON VAUPEL KLEIN, 1996. Mounting media revisited: The suitability of Reyne's fluid for small crustaceans. Crustaceana, 69(6): 794-798. 